\title{
Time-Series Spectroscopy of EC 14026 Stars: Preliminary Results
}

\author{
Simon J. O'Toole
}

Chatterton Astronomy Department, School of Physics, University of Sydney, NSW 2006, Australia

Teresa C. Teixeira

Institute of Physics and Astronomy, University of Aarhus, DK-8000 Aarhus C, Denmark

Timothy R. Bedding

Chatterton Astronomy Department, School of Physics, University of Sydney, NSW 2006, Australia

Hans Kjeldsen

Theoretical Astrophysics Center, University of Aarhus, DK-8000 Aarhus $C$, Denmark

\begin{abstract}
We have obtained time-series spectra for the pulsating hot subdwarf (sdB) PG1605+072. Previous time-series photometry of this star has shown maximum amplitude variations of $\sim 0.1 \mathrm{mag}$ and at least 50 periods. The pulsator has the largest amplitude and longest periods of all the pulsating sdBs (or EC 14026 stars) discovered so far, and appears to be unusual in its class. Preliminary results of a search for velocity variations are presented here. With these variations, along with equivalent width measurements, oscillation mode identification should be possible.
\end{abstract}

\section{Discussion and Further Work}

The main results from our preliminary spectroscopic studies are:

- We have shown the feasibility of using modest size telescopes (such as the Danish 1.54-m) to do time-series spectroscopy of EC 14026 stars.

- Velocity variations in EC 14026 stars have been detected. This confirms that the previous photometric detection of variability is due to stellar pulsation (not that there were many doubts).

- We have obtained 11 nights observations on the Danish 1.54-m telescope and as well as 10 nights on the $1.9-\mathrm{m}$ telescope at Mt Stromlo. With these observations we will have the opportunity to use the complete array of asteroseismological tools to identify oscillation modes, and hence probe the atmospheres of these stars. Analysis of these data will follow. 


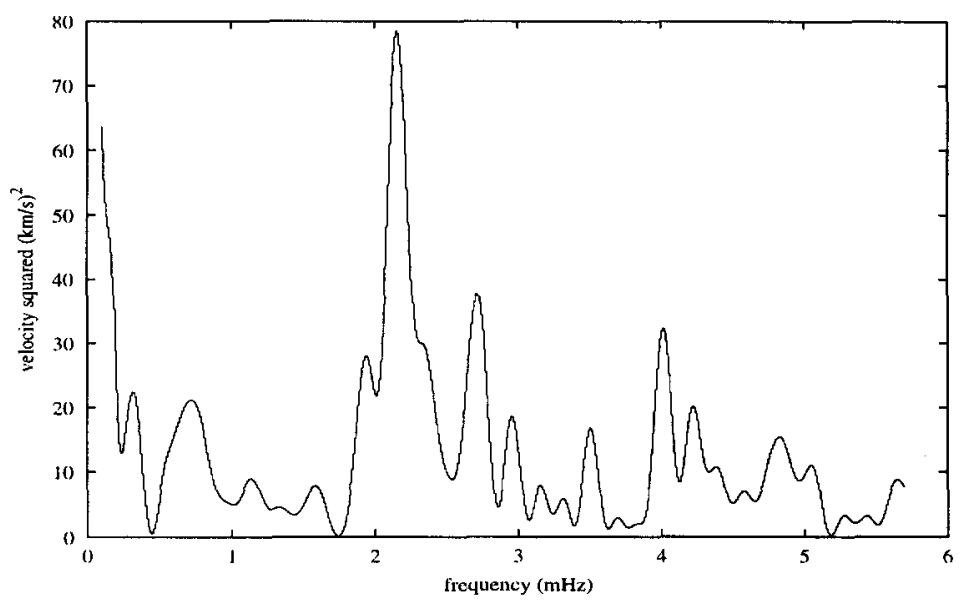

Figure 1. Power spectrum of PG1605+072 using both $\mathrm{H} \beta$ and $\mathrm{H} \gamma$, averaged over two nights. The peak at $\sim 2.10 \mathrm{mHz}$ corresponds to the strongest photometric amplitude found by Koen et al. (1998).

\section{Reference}

Koen, C., O'Donoghue, D., Kilkenny, D., Lynas-Gray, A. E., Marang, F., \& van Wyk, F. 1998, MNRAS, 296, 317 